\title{
A comparison in precision and accuracy of the conventional syringe to the Suh precision syringe
}

This article was published in the following Dove Press journal:

Clinical Ophthalmology

\author{
Devin DeLuna' \\ Audrey Netzel ${ }^{2}$ \\ Jamie Dietze (D) \\ Brett A Begley' \\ Jideofor K Ndulue' \\ Donny W Suh $\mathbb{D}^{\prime}$ \\ 'Department of Ophthalmology, \\ University of Nebraska Medical Center, \\ Omaha, NE, USA; ${ }^{2}$ Department of \\ Ophthalmology, Creighton University \\ School of Medicine, Omaha, NE, USA
}

Correspondence: Audrey Netzel Department of Ophthalmology, Creighton University School of Medicine, 626 N 4l st Street, Omaha, NE 68I3I, USA

Tel + I 6082147857

Email audreynetzel@creighton.edu
Purpose: To compare the accuracy and precision of a conventional intraocular syringe to the Suh precision syringe (SPS), a new injection device designed to decrease operator error and to reduce adverse outcomes by providing a more ergonomic technique for periocular and intraocular injections.

Methods: Participants were instructed to inject a specified quantity of water into a $0.2 \mathrm{~mL}$ polymerase chain reaction (PCR) tube and a sheep eye, first with the conventional syringe, and then with the SPS. The degree of needle movement during injection was video recorded and analyzed for forward-retraction movement and variability in movement. The volume of water injected into the PCR tube was also documented. Data was analyzed using a Wilcoxon signed-rank test.

Results: As compared to the conventional syringe, the SPS had significantly less forwardretraction movement when injecting into the sheep eye $(P=0.04)$. Similarly, the forwardretraction movement of the needle when injecting into the PCR tube was less while using the SPS compared with the conventional syringe; however, this was not statistically significant $(P=0.09)$. There was no significant difference in the volume of water expelled from both syringes $(P=0.28)$.

Conclusion: The SPS demonstrates significant potential to decrease the overall risk involved with injections through reduction of forward-retraction movement during device operation. This new syringe design also provides better control with injection depth and drug injection volume. With its more ergonomic design, the SPS has the potential to decrease risks associated with intraocular injections by improving the accuracy and precision of the injection.

Keywords: Suh precision syringe, intraocular injection

\section{Introduction}

Periocular and intraocular injections are two major means of delivery for a large selection of medications. Each of these injections have their own profiles of indications and risks. For instance, intravitreal delivery of medications is an increasingly common method of treating multiple eye diseases, including retinopathy of prematurity, diabetic retinopathy, age-related macular degeneration, and retinal vein occlusions. ${ }^{1-3}$ Since the introduction of anti-vascular endothelial growth factor (VEGF) medications, intravitreal injections have become a preferred daily practice for numerous ophthalmologists and retina specialists. ${ }^{4-6}$ Additionally, periocular injections are often necessary for many anesthetic procedures and steroid injections. Thus, for many physicians, accuracy and precision when performing these injections is essential to improve patient safety and reduce the potential for adverse outcomes. 
Though complications are rare, the risks and challenges associated with these injections are serious and substantial. The risks of intravitreal injections include pain, bleeding, infection, cataract development, perforation of the retina, and loss of vision. ${ }^{7,8}$ Periocular injections can be associated with risks that include ecchymosis, chemosis, retrobulbar hemorrhage, and more. ${ }^{13}$

Challenges associated with these injections include stabilizing the eyeball before inserting the needle, moving a finger swiftly and stably to press the lever after inserting the needle, injecting precise amounts of small fluid volumes, and removing the needle carefully without damaging any structures. To limit the potential for adverse outcomes and to improve safety and control during periocular and intraocular injections, a prototype of a new onehanded syringe - the Suh precision syringe (SPS) - has been developed at the University of Nebraska Medical Center. This new syringe was designed such that it can be held like a pencil with the barrel held between the thumb and middle finger while the index finger presses on the newly-positioned plunger flange (Figure 1). With the plunger flange placed in a position closer to the neck of the syringe, the user can assume a more ergonomic grip and eliminate the need for an assistant to help deliver the injection. This prospective study tests the idea that the new design improves the overall control - a characteristic measured by forward-retraction movement - and the subsequent precision and accuracy of the injection volume.

\section{Methods and materials}

This study utilized 6 participants all with various levels of medical training: 4 medical students, 1 ophthalmology resident, and 1 pediatric ophthalmologist. The medical students had no prior experience delivering intraocular injections. The resident had previously performed 5 simulated and 3 live intraocular injections. The physician had performed approximately 500 intraocular injections before this time. These values were self-reported. IRB approval or exemption was not required for this study as it does not meet the definition of "human subject" research.

\section{Materials}

The conventional syringe used in the study was a $1 \mathrm{~mL} B D$ (Becton-Dickinson) Tuberculin Slip Tip syringe (REF 309659). The SPS prototype used in the study was constructed using the barrel of another $1 \mathrm{~mL}$ BD Tuberculin Slip-Tip syringe (Figure 1). The needles used in the study were 30-gauge BD PrecisionGlide ${ }^{\mathrm{TM}}$ needles (REF 305106).

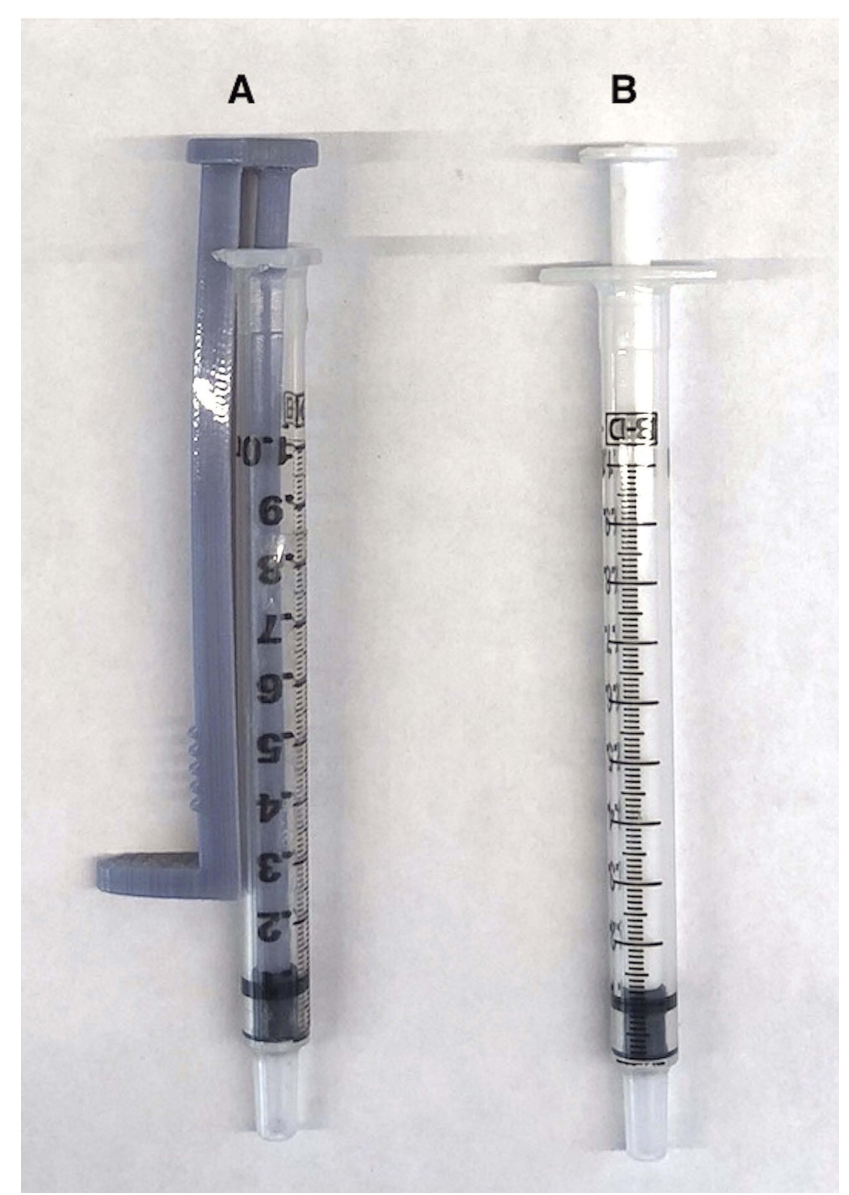

Figure I (A) The Suh precision syringe and (B) a conventional syringe.

The sheep eyes were sourced from Sierra for Medical Science. Ethical approval was not required because no people, medical records, nor human samples were used in the study.

\section{Measurement of injection accuracy \& needle control with analytical model}

Each participant performed two injections with each syringe, precision and conventional. While gloved, the participant used their left hand to place an empty, pre-labeled and pre-weighed $0.2 \mathrm{~mL}$ PCR tube in a tube rack. After aspiration of $0.3 \mathrm{~mL}$ of deionized $\mathrm{H}_{2} \mathrm{O}$, the participant injected a target volume of $0.05 \mathrm{~mL}$ into the tube using their right hand. Following injection, the mass of the tube, now filled with water, was measured again. The mass of the water injected was calculated by taking the difference between the two recorded masses of the tube-with and without water. The volume of water injected was then calculated using the density of water at the temperature 
of the room. A hand rest was provided and used by each participant during injection. For each participant, this process was first completed using the conventional syringe and followed by the precision syringe. The results from the conventional and precision syringe were compared using a Wilcoxon signed-rank test. While participants performed this task, video footage was recorded to analyze hand movement while performing the injection. See VideoBased Measurement of Needle Control.

\section{Measurement of needle control with patient model}

A patient model was created to test the precision syringe against the conventional syringe. The model was constructed using a foam mold of a human head with an insert for an eye. A sheep eye was placed in the insert. Each participant performed one injection using the precision and conventional syringe. With the same technique used in the analytical model, video footage was recorded and analyzed while each injection was performed. See Video-Based Measurement of Needle Control.

\section{Video-based measurement of needle control}

A video-based method of measuring needle control was used to compare the precision and conventional syringes while performing the procedures discussed previously. All injections were recorded using two camcorders. Camcorder 1 (Panasonic HC-VX1 4K) was positioned facing the participant and tube rack or sheep eye. This camcorder was placed approximately $7 \mathrm{~cm}$ away and focused on the PCR tube or sheep eye. To capture larger hand movements, camcorder 2 (Panasonic AG-UX90) - at a right angle to camcorder 1 -was positioned approximately 4 feet to the left of the participant and tube rack or sheep eye. The tube rack and both cameras were fixed in place to avoid movement during the experiment.

The footage was analyzed using Adobe Premiere Pro CC. The footage was imported as an mp4 file with a frame rate of 59.94 frames per second (fps). Focus was placed on measuring vertical movement of the syringe and needle. Movements in the horizontal plane were ignored since this movement is largely restricted by the tissue of the eye during a real injection. Vertical movement of the needle during each injection was evaluated over the time that the participant applied pressure to the plunger. For the analytical model, this time was standardized by designating the start of time as the frame in which the first droplet of water detached from the needleas a result of the pressure applied to the plunger - and began to fall into the tube. The point at which the participant stopped applying pressure to the plunger was approximated by setting the end of time as the frame in which the droplet of water emerging from the needle stopped enlarging. The time during which vertical movement was analyzed in the patient model was standardized in a different manner due to inability to visualize the fluid emerging from the tip of the needle. Specifically, the start of time was set as the video frame in which the surface of sclera surrounding the puncture site had no longer experienced downward depression due to the needle - a moment approximating the time in which the needle fully punctured the sclera. Because the tip of the needle was not visible, the vertical movement of the needle was tracked by watching a fixed point at the base of the needle. The end of time during which movement was analyzed was set as the frame immediately before deliberate movement was made to pull the needle out of the eye. Deliberate movement was defined as the frame preceding consecutive frames of rapid movement away from the surface of the eye leading to the exit from the tissue.

For every injection, measurement of the vertical movement was done by identifying the frame in which the needle was at its highest point (i.e. retracted movement) and the frame in which the needle was at its lowest point (i.e. forward movement). These two frames were both exported to Adobe Photoshop as JPEG files. The two frames were superimposed on one another as separate layers in Photoshop. The transparency of the top layer was increased to simultaneously visualize the needle at its high point and low point. For the analytical model, grid lines were placed at the tip of the needle at both points in time. For injections performed on the sheep eyes, grid lines were placed at the base of the needle since the tip was not visible. The difference in height between the low point and high point of the superimposed frames was measured by finding the vertical distance, in pixels, between the gridlines (Figure 2) using the electronic ruler tool in photoshop (not depicted). A conversion factor was used to convert pixels to millimeters. This was done by using a caliper to measure the length of the needle in-person in millimeters and via Photoshop using the ruler tool. This conversion factor had to be calculated for both the analytical model and patient model because the position of the camcorders were adjusted slightly. The data for vertical displacement of the precision and conventional 


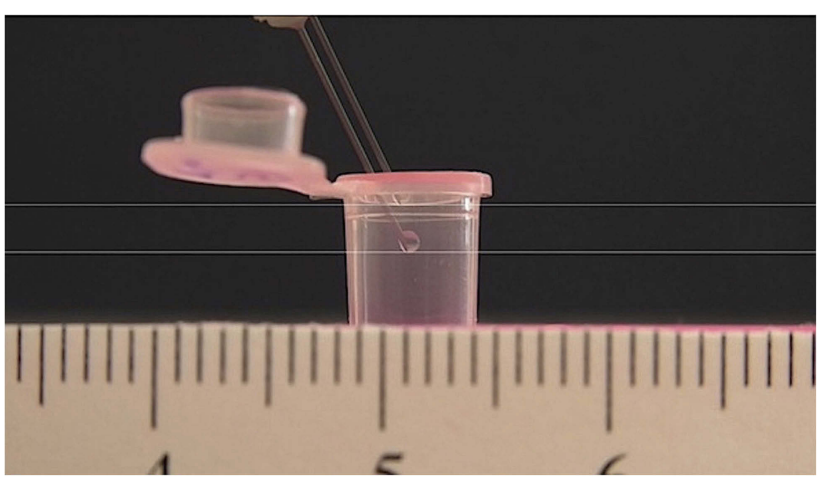

Figure 2 Demonstration of the ruler tool used to calculate the forward-retraction movement of needles.

syringe were compared using a Wilcoxon signed-rank test and box plot.

\section{Data analysis}

Parameters compared were (1) forward-retraction movement of the needle during injection into a $0.2 \mathrm{~mL}$ PCR tube and sheep eye, (2) variability in forward-retraction movement between first and second attempts at injection into $0.2 \mathrm{~mL}$ PCR tube, and (3) volume of water expelled from the syringe. This data is recorded in Table 1. Normality of data was evaluated by plotting a histogram and then overlaying a normal distribution curve. The Wilcoxon signed-rank test was performed to assess for differences in median values between the precision and conventional syringe. It is the most suitable analysis for the data because the data was not normally distributed and the precision and conventional syringes were used twice by the same individuals. Box-whisker plots were created for forward-retraction movement during injection into the PCR tube (Figure 3 ) and into a sheep eye (Figure 4). A $P$-value of 0.05 was considered as statistically significant.

\section{Results}

Compared with the conventional syringe, the SPS had significantly less forward-retraction movement when injecting into a sheep eye (SPS v. conventional; $3.53 \mathrm{~mm} \mathrm{v.} 4.46 \mathrm{~mm}$, $P=0.04)$. Similarly, the forward-retraction movement of the needle when injecting into the PCR tube was less while using the SPS compared with the conventional syringe; however, this was not statistically significant (precision v. conventional; $2.35 \mathrm{~mm}$ v. $2.85 \mathrm{~mm}, P=0.09$ ). The precision syringe also had less variability in the forward-retraction movement between the first and second attempts at injecting into the PCR tube, but was not statistically significant $(P=0.68)$. There was no significant difference $(P=0.28)$ in the volume of water expelled from both syringes.

\section{Discussion}

The proper execution of intraocular and periocular injections is important to avoid the risks associated with the procedures. Successful injection relies on careful placement of the injection site and control of the needle during the injection. This requires the provider to deliver a controlled injection while also stabilizing the patient's eye - a task that sometimes requires two individuals. This can become particularly difficult for the pediatric ophthalmologist whose patients do not always cooperate during such a controlled procedure. Infants are a high-risk group for injections due to an underdeveloped pars plana and short anteroposterior globe diameter. This easily allows for iatrogenic breaks in the anterior retina at the time of injection. ${ }^{9}$ The size of the pars plana can be difficult to estimate based on the inability of direct visualization. Even measurements of the pars plana given in reports have varied. . Additionally, an infant's lens is large in relation to the neonatal globe, allowing it to be vulnerable to injury if the angle of the injection is not parallel to the visual axis. ${ }^{9}$ Not only are there anatomical factors making an injection difficult, but the unpredictability of infant cooperation also plays a major role. Even once an injection is made, there is still a higher risk for complication due to an infant's underdeveloped immune system predisposing them to a higher risk of infection. ${ }^{9}$ Previous studies have

Table I Comparison between conventional and Suh precision syringe. Comparisons were made using Wilcoxon signed-rank test

\begin{tabular}{|c|c|c|c|}
\hline \multirow[t]{2}{*}{ Variables } & Conventional Syringe & Suh Precision Syringe & \multirow[t]{2}{*}{ P-value } \\
\hline & Median (QI; Q3) & Median (QI; Q3) & \\
\hline Forward-retraction movement ${ }^{\mathrm{a}}(\mathrm{mm})$ & $2.85(2.30 ; 3.10)$ & $2.35(2.10 ; 3.10)$ & 0.09 \\
\hline Variability in forward-retraction movement ${ }^{\mathrm{a}}$ between Ist and 2 nd attempt & $0.60(0.30 ; 1.50)$ & $0.50(-1.00 ; 1.00)$ & 0.68 \\
\hline Forward-retraction movement ${ }^{\mathrm{b}}(\mathrm{mm})$ & $4.46(3.35 ; 4.62)$ & $3.53(3.23 ; 4.42)$ & $0.04 *$ \\
\hline Water expelled from syringe $(\mathrm{mL})$ & $0.05(0.04 ; 0.05)$ & $0.05(0.05 ; 0.05)$ & 0.28 \\
\hline
\end{tabular}

Notes: $\mathrm{QI}=25$ th percentile; $\mathrm{Q} 3=75$ th percentile. ${ }^{\mathrm{a}}$ Injection into a $0.2 \mathrm{~mL}$ tube. ${ }^{\mathrm{b}}$ Injection into sheep eye. ${ }^{*}$ Indicates statistical significance with $P<0.05$. 


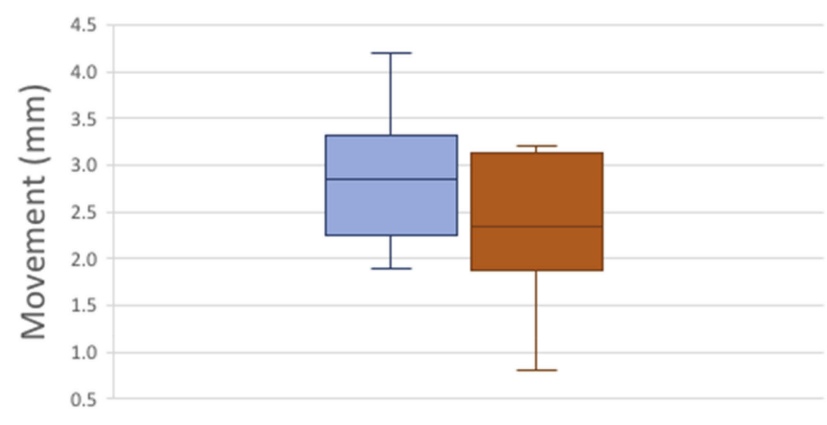

$\square$ Conventional Syringe $\square$ Suh Precision Syringe

Figure 3 Box plot comparing forward-retraction movement of a conventional syringe and the SPS during injection of water into a $0.2 \mathrm{~mL}$ PCR tube.

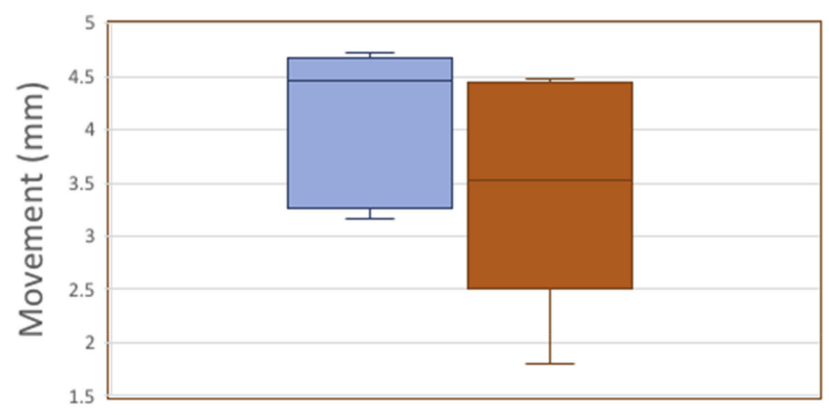

$\square$ Conventional Syringe $\square$ Suh Precision Syringe

Figure 4 Box plot comparing forward-retraction movement between a conventional syringe and the SPS while injecting water into a sheep eye.

suggested that the best way to overcome some of the obstacles mentioned here are to use a short needle and a small volume syringe for good hand control and less risk of perforation. ${ }^{9}$ The needle tip must be kept parallel with the pupillary axis and administered just posterior to the temporal limbus to avoid injury. ${ }^{9}$

While there are patient factors posing risks, there are provider factors as well. Provider skill level of precision and accuracy in injection plays a major role. One component of the provider's skill level is in the tools available for use. A study looking at the ergonomics of dental syringes was performed and concluded that a petite syringe allowed for significant improvement in administration and overall feeling of control. ${ }^{10}$ Other studies have looked at the ergonomics behind the different angles between the thumb and index finger with the force needed to produce movement. With a larger distance between the thumb and index finger, more force is needed to move the same amount of weight. ${ }^{11}$ These concepts of better ergonomic control for the administrator was the rationale behind our precision syringe device.
Thus, in keeping with the recommended use of a short needle and small volume syringe as well as the optimal angles for dexterity, the precision syringe design was developed. The design brings the index finger closer to the thumb and middle finger to allow for better stabilization and control during injection. This better control was shown in our trial of injection into a sheep eye model where there was a difference of $0.97 \mathrm{~mm}$ between the median values of the trial comparing forward-retraction movement in the conventional syringe and the SPS. Compared with the conventional syringe, the SPS showed better control while injecting into the sheep eye model. This was evidenced by significantly less forward-retraction movement $(0.97 \mathrm{~mm} ; P=0.04)$ during the injection. This decrease of $0.97 \mathrm{~mm}$ of variability in injection placement would make a significant clinical difference as even a 1month-old infant only has a $2 \mathrm{~mm}$ pars plana ${ }^{12}$ through which intravitreal injections occur and a movement of almost $1 \mathrm{~mm}$ could easily disrupt the vitreous and retinal structures. While the forward-retraction movement was not shown to be decreased significantly in the test tube model, the median movement was still decreased. The variation can be attributed to less stability as there was no surrounding structures to help anchor the user's hand while performing the technique and there was no surrounding injection material to help provide movement resistance as would be seen with a true injection. The sheep eye model demonstrates a more accurate depiction of a real injection and should more accurately reflect what happens in an intraocular injection.

While variation in hand size was not measured, it can be hypothesized that those with smaller hands will find the precision syringe design more comfortable to use, as was found in the previously mentioned dental study by Wiener et al. ${ }^{10}$ Another area of note is that by moving the plunger flange down closer to the needle tip, the operator can better visualize the movement of the needle tip and the amount of finger movement, which provides better feedback for users to refine their hand-eye coordination in future injections.

Another consideration to be made is the level of injection experience among users. While injecting into the PCR tube using the precision syringe, the pediatric ophthalmologist demonstrated decreased forward-retraction movement and a more accurate injected water volume (Table 2). This trend is particularly promising because, with further research, it may indicate that syringe users already experienced in conventional syringe injection may easily transition to the SPS to 
Table 2 Injections into a $0.2 \mathrm{~mL}$ PCR tube by a practicing pediatric ophthalmologist

\begin{tabular}{|l|l|l|}
\hline Variables & Conventional Syringe & Suh Precision Syringe \\
\hline Average forward-retraction movement $(\mathrm{mm})$ & 2.5 & 1.85 \\
Average water expelled from syringe $(\mathrm{mL})$ & 0.031 & 0.052 \\
\hline
\end{tabular}

achieve greater accuracy and precision during injections. Another contributing factor to the improved outcomes for the experienced user may be greater finger dexterity and strength attained through many years of delicate hand movements during injections and eye surgeries. Future studies observing a larger group of individuals experienced in intraocular injections will more clearly elucidate this trend.

While this study did not find a statistical difference in the amount of water dispensed by the precision syringe as compared to the conventional syringe, users may have become more precise when using the Suh syringe (Table 1; precision v. conventional; 0.005 v. 0.011). This improved precision is critical to successfully administering intraocular injections. Results show that users became more consistent at administering an inaccurate quantity of water with the SPS. During the experiment, users commented that lines on the syringe designating quantity of water were not easily visible and partially covered by the Suh plunger during injections. While this observation may contribute to the decline in accuracy seen with the precision syringe, it is also a design element that can be remedied in future syringe prototypes.

Greater control of the syringe during the injection process is especially noteworthy during injections with infants, many are crying and wiggling, making proper technique difficult to perform. By allowing an additional stabilization point during injections and an extra force to help steady infant head movements, the precision syringe theoretically makes significant contributions for easily accomplishing proper injection technique. Another theoretical benefit of the SPS is that the user can always visualize the needle tip during the injection and never needs to glance away to ensure proper finger placement on the syringe plunger, as would be required in the standard syringe. This potentially lowers the risk of an accidental needle stick.

This study is limited in a few aspects. The first limitation is the small number of participants and low quantity of repetitions made with each syringe. The small sample size makes it difficult to perform a meaningful statistical analysis. However, this is the reason the normality of the data was evaluated by plotting a histogram and overlaying a normal distribution curve. Future studies should utilize a larger number of participants and ideally obtain 5 or more repetitions with each syringe to match that required during calibration of pipettes which aliquot identical volumes of liquid. Limitations also exist in the ability to measure syringe movement. This study was limited to analyzing one variable to assess control of the syringe: anteroposterior, or forward-retraction, movement. Only one plane of movement was analyzed due to three constraints. The first constraint is the time intensive process of data collection attributed with analyzing video at 60 frames per second. The second constraint is establishing standardized time intervals during which the syringe displacement is measured. This is particularly difficult while the syringe is in free space above the injection site - a time during which it is not always possible to differentiate between intentional and unintentional movement. Third, during injection, the needle is limited to movement in one plane due to restraint by the surrounding tissues. Due to these issues, forwardretraction movement is one of the few objective variables that could be used to measure the overall control of the syringe. Ideally, future studies should analyze movement of the syringe in multiple planes of movement, perhaps using computer software and additional camera angles.

\section{Conclusion}

From an operator standpoint, the Suh precision syringe proves to have the potential to decrease the overall risk involved with injections through reduction of forwardretraction movement, and thus, improved control, during device operation. Placement of the syringe plunger closer to the needle tip, as designed in the SPS, allows the operator to better visualize needle tip movement and eliminates the need to glance away from the needle in order to ensure proper finger placement on the syringe plunger during an injection. By allowing the user to have more control with the injection depth and drug injection volume through better stabilization and optimal ergonomics, there is less risk of damage to the lens or lens capsule, corneal abrasion, intraocular hemorrhage, or retinal tears. While 
this new design cannot take away all the risk of potential iatrogenic injury with injections, it does show promise to help decrease operator error by giving a more ergonomic method for better precision and accuracy.

\section{Disclosure}

Donny W Suh owns the patent for the Suh precision syringe. The authors report no other conflicts of interest in this work.

\section{References}

1. Cheung N, Wong IY, Wong TY. Ocular anti-VEGF therapy for diabetic retinopathy: overview of clinical efficacy and evolving applications. Diabetes Care. 2014;37(4):900-905. doi:10.2337/dc13-1990

2. Ba J, Peng R, Xu D, et al. Intravitreal anti-VEGF injections for treating wet age-related macular degeneration: a systematic review and meta-analysis. Drug Des Devel Ther. 2015;9:5397-5405. doi:10.2147/DDDT.S86269

3. Rezar S, Eibenberger K, Bühl W, Georgopoulos M, Schmidt-Erfurth U, Sacu S, Macula Study Group Vienna. Anti-VEGF treatment in branch retinal vein occlusion: a real-world experience over 4 years. Acta Ophthalmol. 2015;93(8):719-725. doi:10.1111/aos.12772

4. American Academy of Ophthalmology Retina/Vitreous Panel. Preferred Practice Pattern Guidelines. Diabetic Retinopathy. San Francisco, CA: American Academy of Ophthalmology;2017. Availavle from: http:// www.aao.org/ppp. Accessed August 13, 2018.
5. American Academy of Ophthalmology Retina/Vitreous Panel. Preferred Practice Pattern Guidelines. Age-Related Macular Degeneration. San Francisco, CA: American Academy of Ophthalmology;2015. Availavle from:. http://www.aao.org/ppp. Accessed August 13, 2018.

6. American Academy of Ophthalmology Retina/Vitreous Panel. Preferred Practice Pattern Guidelines. Retinal Vein Occlusions. San Francisco, CA: American Academy of Ophthalmology;2015. Availavle from: http://www.aao.org/ppp. Accessed August 13, 2018.

7. Tolentino M. Systemic and ocular safety of intravitreal anti-VEGF therapies for ocular neovascular disease. Surv Ophthalmol. 2011;56 (2):95-113. doi:10.1016/j.survophthal.2010.08.006

8. Ghasemi KF, Nguyen QD. Adverse events and complications associated with intravitreal injection of anti-VEGF agents: a review of literature. Eye. 2013;27:787-794. doi:10.1038/eye.2013.107

9. Wright LM, Vrcek IM, Scribbick FW III, Chang EY, Harper CA III. Technique for infant intravitreal injection in treatment of retinopathy of prematurity. Retina. 2017;37(11):2188-2190. doi:10.1097/IAE.000000 0000001561

10. Wiener RC, Crout RJ, Sandell J, et al. Local anesthetic syringe ergonomics and student preferences. Journal of Dental Education. 2009;73(4):518-522.

11. Li K, Nataraj R, Marquardt TL, Li ZM. Directional coordination of thumb and finger forces during precision punch. PLoS One. 2013;8 (11):e79400. doi:10.1371/journal.pone.0079400

12. Hairston RJ, Maguire AM, Vitale S, Green WR. Morphometric analysis of pars plana development in humans. Retina. 1997;17(2):135-138.

13. Rubin AP. Complications of local anaesthesia for ophthalmic surgery. British Journal of Anaesthesia. 1995;75:93-96. doi:10.1093/bja/ 75.1.93
Clinical Ophthalmology

\section{Publish your work in this journal}

Clinical Ophthalmology is an international, peer-reviewed journal covering all subspecialties within ophthalmology. Key topics include: Optometry; Visual science; Pharmacology and drug therapy in eye diseases; Basic Sciences; Primary and Secondary eye care; Patient Safety and Quality of Care Improvements. This journal is indexed on PubMed

\section{Dovepress}

Central and CAS, and is the official journal of The Society of Clinical Ophthalmology (SCO). The manuscript management system is completely online and includes a very quick and fair peer-review system, which is all easy to use. Visit http://www.dovepress.com/ testimonials.php to read real quotes from published authors. 